\title{
Dark Matter Has Already Been Discovered
}

\author{
Koshun Suto ${ }^{1}$ \\ ${ }^{1}$ Chudai-ji Temple, Isesaki, Japan \\ Correspondence: Koshun Suto, Chudai-Ji Temple, 5-24, Oote-Town, Isesaki, 372-0048, Japan. E-mail: \\ koshun_suto129@mbr.nifty.com
}

Received: December 8, 2021

Accepted: January 6, 2022

Online Published: January 20, 2022

doi:10.5539/apr.v13n3p36

URL: https://doi.org/10.5539/apr.v13n3p36

\begin{abstract}
The energy-momentum relationship in the special theory of relativity (STR) holds in an isolated system in free space. However, this relationship is not applicable to an electron in a hydrogen atom where there is potential energy. Using three types of methods, the author has already derived an energy-momentum relationship applicable to an electron in a hydrogen atom. In the past, Dirac asserted that Einstein's relationship has negative solutions. This paper too obtains negative solutions (energy) from the derived relationship using Dirac's reasoning. However, the discontinuity peculiar to the micro world is not incorporated into that solution. Thus discontinuity is incorporated into the solution by using a new quantum condition already derived by the author. Next, the orbital radius of an electron with negative energy in an absolute sense is found, and that radius is compared with the orbital radius of an electron in an ordinary hydrogen atom. A search is conducted for experiments supporting the DM model advocated by this paper. A hydrogen atom at this ultra-low energy level is formed from an atomic nucleus (proton) with positive mass, and a single electron with negative mass existing near that. In this paper, this unknown type of matter will be called a dark hydrogen atom (DHA). This paper also points out that DHA accounts for part of the true nature of dark matter (DM), the mysterious material whose true nature is currently unknown. Although this differs from the conventional interpretation, this paper holds that the experiment which demonstrates the existence of DHA is triplet production. The paper points out that one of the two electrons produced in a triplet production experiment is the electron which forms a DHA. If the DM model advocated by this paper is correct, then DM has already been discovered.
\end{abstract}

Keywords: dark matter, Einstein's energy-momentum relationship, ultra-low energy levels in the hydrogen atom, dark hydrogen atom, triplet production

\section{Introduction}

The existence of dark matter (DM), a mysterious material which exists in space, was first noticed by the astronomer Fritz Zwicky (Zwicky, 1933). In the 1930s, he observed groups of galaxies called galaxy clusters. At that time, he noticed that the galaxies of the Coma Cluster were moving at a speed sufficient to fly away from the cluster. Nevertheless, the galaxy cluster did not fly apart. Zwicky predicted that something is present, besides visible matter, which increases the gravity of the galaxy cluster. In 1933, Zwicky named this something "missing mass" and recognized its importance. This was a revolutionary theory, but no one paid attention to his hypothesis at the time. At that time, full-fledged observation of space had just begun. It was not possible to determine whether this unknown type of matter was just invisible galaxies, or gas, or something completely different. Scientists first began paying attention to Zwicky's claims 40 years later.

In the 1970s, the astronomer Vera Rubin observed galaxies and discovered that the outside of a galaxy has the same rotation speed as the inside. According to Newtonian mechanics, the rotation speed of the outside of the galaxy should be slower than the rotation speed of the inside. In fact, in the case of the solar system, planets on the inside rotate faster than planets on the outside. Rubin concluded that something invisible with a large mass acts as a gravitational source and affects the rotation of a galaxy. After that, astronomers all over the world began verifying these observations.

The first candidates for DM, this unknown matter, were dark, invisible objects, neutrinos, black holes, and so forth. However, these candidates were ruled out by experiments.

In the 21st century, WIMPs and Axions became leading candidates, and experiments to detect them are being conducted. However, news of the discovery of DM has still not arrived. 
This paper describes the DM candidate advocated by the author. The steps of the paper are as follows:

1. An energy-momentum relationship applicable to an electron in a hydrogen atom is derived.

2. In the past, Dirac asserted that Einstein's relationship has negative solutions. This paper too obtains negative solutions (energy) from the derived relationship using Dirac's reasoning.

3. The discontinuity peculiar to the micro world is not incorporated into that solution. Thus discontinuity is incorporated into the solution by using a new quantum condition already derived by the author.

4. Next, the orbital radius of an electron with negative energy in an absolute sense is found, and that radius is compared with the orbital radius of an electron in an ordinary hydrogen atom.

5. A search is conducted for experiments supporting the DM model advocated by this paper.

\section{Energy-Momentum Relationship Applicable to an Electron in a Hydrogen Atom}

The energy-momentum relationship in the special theory of relativity (STR) holds in an isolated system in free space. Here, if $m_{0} c^{2}$ is the rest mass energy and $m c^{2}$ is the relativistic energy, the relationship can be written as follows (Einstein, 1961).

$$
\left(m_{0} c^{2}\right)^{2}+p^{2} c^{2}=\left(m c^{2}\right)^{2} .
$$

This relationship is not applicable to an electron in a hydrogen atom where there is potential energy. Therefore, from the beginning we should have looked for an energy-momentum relationship applicable to an electron in a hydrogen atom. However, there was no search for such a relationship.

This is because it has been thought that quantum mechanics is the theory describing the behavior of an electron in a hydrogen atom.

Using three types of methods, the author has already derived an energy-momentum relationship applicable to an electron in a hydrogen atom (Suto, 2011; Suto, 2020a; Suto, 2020b). This paper presents the method derived in the second paper.

Now, the following equation holds due to Formula (1).

$$
\left(m c^{2}\right)^{2}=m_{0}^{2} c^{4}+\left(m^{2} c^{4}-m_{0}^{2} c^{4}\right)=\left(m_{0} c^{2}\right)^{2}+\left(m+m_{0}\right)\left(m c^{2}-m_{0} c^{2}\right) c^{2} .
$$

Using this, Formula (1) becomes as follows.

$$
\left(m_{0} c^{2}\right)^{2}+\left(m+m_{0}\right)\left(m c^{2}-m_{0} c^{2}\right) c^{2}=\left(m c^{2}\right)^{2} .
$$

Incidentally, Sommerfeld and Einstein defined relativistic kinetic energy as follows (Sommerfeld, 1923).

$$
K_{\mathrm{re}}=m c^{2}-m_{0} c^{2} .
$$

Since Formulas (1) and (3) are equal, the following relationship must hold when Formula (4) is taken into account.

$$
p^{2}=\left(m_{0}+m\right)\left(m c^{2}-m_{0} c^{2}\right)=\left(m_{0}+m\right) K_{\mathrm{re}} .
$$

The following formula is obtained from this.

$$
K_{\mathrm{re}}=\frac{p^{2}}{m_{0}+m}, \quad p=m_{0} v .
$$

Next, the relativistic kinetic energy of an electron in a hydrogen atom is defined as follows by referring to Formula (6).

$$
K_{\mathrm{re}, n}=\frac{p_{\mathrm{re}, n}^{2}}{m_{\mathrm{e}}+m_{n}}, \quad p_{\mathrm{re}, n}=m_{n} v_{n} .
$$


Here, $m_{n}$ is the relativistic mass of the electron. Also, $p_{\mathrm{re}, n}$ indicates the relativistic momentum of the electron.

Incidentally, the energy of an electron at rest in an isolated system in free space is $m_{\mathrm{e}} c^{2}$. Here, we consider the case where this electron is drawn in by the electrical attraction of the proton, and forms a hydrogen atom. At this time, the electron emits a photon to the outside. Therefore, the relativistic energy of an electron in a hydrogen atom $m_{n} c^{2}$ becomes smaller than the rest mass energy $m_{\mathrm{e}} c^{2}$. That is,

$$
m_{n} c^{2}<m_{\mathrm{e}} c^{2}
$$

The behavior of an electron inside an atom, where there is potential energy, cannot be described with the relationship of Einstein (1). Caution is necessary because it is completely overlooked in Formula (8).

Sommerfeld thought the kinetic energy of an electron in a hydrogen atom could be described with Formula (4). However, this is an error.

Now, referring to Formula (4), it is natural to define the relativistic kinetic energy of an electron in a hydrogen atom as follows (Suto, 2019).

$$
K_{\mathrm{re}, n}=-E_{\mathrm{re}, n}=m_{\mathrm{e}} c^{2}-m_{n} c^{2}
$$

This paper defines $E_{\mathrm{re}, n}$ as the relativistic energy levels of the hydrogen atom derived at the level of classical

quantum theory. (The quantum number used here is just the principal quantum number. Therefore, $E_{\mathrm{re}, n}$ is not a formula which predicts all the relativistic energy levels of the hydrogen atom.)

However, the term "relativistic" used here does not mean based on the STR. It means that the expression takes into account the fact that the mass of the electron varies due to velocity. According to the STR, the electron's mass increases when its velocity increases. However, inside the hydrogen atom, the mass of the electron decreases when the velocity of the electron increases. Attention must be paid to the fact that, inside the hydrogen atom, the relativistic mass of the electron $m_{n}$ is smaller than the rest mass $m_{\mathrm{e}}$.

In this way, two formulas have been obtained for the relativistic kinetic energy of the electron in a hydrogen atom (Formulas (7), and (9)). Incidentally, the following equation can be derived from Formulas (7) and (9).

$$
\frac{p_{\mathrm{re}, n}^{2}}{m_{\mathrm{e}}+m_{n}}=m_{\mathrm{e}} c^{2}-m_{n} c^{2} .
$$

Rearranging this, the following relationship can be derived.

$$
\left(m_{n} c^{2}\right)^{2}+p_{\mathrm{re}, n}^{2} c^{2}=\left(m_{\mathrm{e}} c^{2}\right)^{2}
$$

Formula (11) is the energy-momentum relationship applicable to the electron in a hydrogen atom.

Now, in the past, Dirac derived the following negative solution from Formula (1).

$$
E=m c^{2}= \pm m_{0} c^{2}\left(1-\frac{v^{2}}{c^{2}}\right)^{-1 / 2}
$$

If the same logic is applied to Formula (11), then the following formula can be derived. 


$$
E= \pm m_{\mathrm{e}} c^{2}\left(1+\frac{v_{n}^{2}}{c^{2}}\right)^{-1 / 2} .
$$

However, Formula (13) does not incorporate the discontinuity peculiar to the micro world. Therefore, Formula (13) must be rewritten into a relationship where energy is discontinuous.

\section{A New Quantum Condition for Incorporating Discontinuity in Formula (13)}

This quantum condition has already been derived in another paper (Suto, 2021).

Incidentally, the energy levels derived by Bohr are given by the following formulas (Bohr, 1913: Bohr, 1952).

$$
E_{\mathrm{BO}, n}=-\frac{1}{2}\left(\frac{1}{4 \pi \varepsilon_{0}}\right)^{2} \frac{m_{\mathrm{e}} e^{4}}{\hbar^{2}} \cdot \frac{1}{n^{2}}, \quad n=1,2, \cdots
$$

Here, $E_{\mathrm{BO}, n}$ signifies the energy levels derived by Bohr.

However, Formula (14) cannot be regarded as beautiful. Intrinsically, the electron has a rest mass energy of $m_{\mathrm{e}} c^{2}$. In Formula (14), the relationship with the rest mass energy of an electron is not clear.

Thus, Formula (14) is rewritten as follows.

$$
E_{\mathrm{BO}, n}=-\frac{\alpha^{2} m_{\mathrm{e}} c^{2}}{2 n^{2}}, \quad n=1,2, \cdots
$$

Here, $\alpha$ is the following fine-structure constant.

$$
\alpha=\frac{e^{2}}{4 \pi \varepsilon_{0} \hbar c} .
$$

From the author's perspective, Formula (15) containing $m_{\mathrm{e}} c^{2}$ is superior to Formula (14) from a physical standpoint.

Also, Bohr's orbital radius $r_{\mathrm{BO}, n}$ is normally described with the following formula.

$$
r_{\mathrm{BO}, n}=4 \pi \varepsilon_{0} \frac{\hbar^{2}}{m_{\mathrm{e}} e^{2}} n^{2} .
$$

However, Formula (17) is not very impressive. This is because, in Formula (17), the relationship between the electron's orbital radius and the classical electron radius $r_{\mathrm{e}}$ is not clear.

Bohr thought the following quantum condition was necessary to find the energy levels of the hydrogen atom.

$$
m_{\mathrm{e}} v_{n} \cdot 2 \pi r_{\mathrm{BO}, n}=2 \pi n \hbar .
$$

In Bohr's theory, the energy levels of the hydrogen atom is treated non-relativistically, and thus here the momentum of the electron is taken to be $m_{\mathrm{e}} \mathrm{v}$. Also, the Planck constant $h$ can be written as follows (Suto, 2020c).

$$
\hbar=\frac{h}{2 \pi}=\frac{m_{\mathrm{e}} c \lambda_{\mathrm{C}}}{2 \pi} .
$$

$\lambda_{\mathrm{C}}$ is the Compton wavelength of the electron.

When Formula (19) is used, the fine-structure constant $\alpha$ can be expressed as follows.

$$
\alpha=\frac{e^{2}}{4 \pi \varepsilon_{0} \hbar c}=\frac{e^{2}}{2 \varepsilon_{0} m_{\mathrm{e}} c^{2} \lambda_{\mathrm{C}}} .
$$

Also, the classical electron radius $r_{\mathrm{e}}$ is defined as follows.

$$
r_{\mathrm{e}}=\frac{e^{2}}{4 \pi \varepsilon_{0} m_{\mathrm{e}} c^{2}} .
$$


If $r_{\mathrm{e}} / \alpha$ is calculated here,

$$
\frac{r_{\mathrm{e}}}{\alpha}=\frac{\lambda_{\mathrm{C}}}{2 \pi} .
$$

If Formula (17) is written using $r_{\mathrm{e}}$ and $\alpha$, the result is as follows.

$$
r_{\mathrm{BO}, n}=4 \pi \varepsilon_{0} \frac{\hbar^{2}}{m_{\mathrm{e}} e^{2}} n^{2}=\frac{e^{2}}{4 \pi \varepsilon_{0} m_{\mathrm{e}} c^{2}}\left(\frac{4 \pi \varepsilon_{0} \hbar c}{e^{2}}\right)^{2} n^{2}=\frac{r_{\mathrm{e}}}{\alpha^{2}} n^{2} .
$$

Formula (23) containing $r_{\mathrm{e}}$ is superior to Formula (17) from a physical standpoint.

Next, if $\hbar$ in Formula (19) and $r_{\mathrm{B} O, n}$ in Formula (23) are substituted into Formula (18),

$$
m_{\mathrm{e}} v_{n} \cdot 2 \pi \frac{r_{\mathrm{e}}}{\alpha^{2}} n^{2}=2 \pi n \frac{m_{\mathrm{e}} c \lambda_{\mathrm{C}}}{2 \pi} .
$$

If Formula (22) is also used, then Formula (24) can be written as follows.

$$
m_{\mathrm{e}} v_{n} \cdot 2 \pi \frac{r_{\mathrm{e}}}{\alpha^{2}} n^{2}=2 \pi n \frac{m_{\mathrm{e}} c r_{\mathrm{e}}}{\alpha} .
$$

From this, the following relationship can be derived.

$$
\frac{v_{n}}{c}=\frac{\alpha}{n}
$$

Due to Formula (26), it is possible to identify discontinuous states that are permissible in terms of quantum mechanics in the continuous motions of classical theory.

\section{Relativistic Energy Levels of the Hydrogen Atom}

If Formula (26) derived in Section 3 is substituted into Formula (13), the relativistic energy $E$ of an electron becomes as follows.

$$
E= \pm m_{\mathrm{e}} c^{2}\left(1+\frac{\alpha^{2}}{n^{2}}\right)^{-1 / 2}
$$

This $E$ is defined as follows.

$$
E_{\mathrm{ab}, n}^{ \pm}=m_{n}^{ \pm} c^{2}= \pm m_{\mathrm{e}} c^{2} \pm E_{\mathrm{re}, n}^{ \pm}= \pm m_{\mathrm{e}} c^{2}\left(1+\frac{\alpha^{2}}{n^{2}}\right)^{-1 / 2} .
$$

$E_{\mathrm{ab}, n}$ gives the relativistic energy of the electron, but this is also the absolute energy of the electron. The "ab" subscript of $E_{\mathrm{ab}, n}$ stands for "absolute." Also, $m_{n} c^{2}$ is the relativistic energy of the electron when the principal quantum number is in the state $n$.

The positive solution of Formula (28), i.e., the relativistic energy levels of an ordinary hydrogen atom, can be expressed as follows. (Ordinarily, there is no problem in omitting the + of $E_{\mathrm{re}, n}^{+}$. Note the difference between the relativistic energy levels of the hydrogen atom $E_{\mathrm{r}, n}$ and the relativistic energy of the electron $E_{\mathrm{ab}, n}$.)

$$
\begin{aligned}
& E_{\mathrm{re}, n}=m_{\mathrm{e}} c^{2}\left[\left(1+\frac{\alpha^{2}}{n^{2}}\right)^{-1 / 2}-1\right] \\
= & m_{\mathrm{e}} c^{2}\left[\left(\frac{n^{2}}{n^{2}+\alpha^{2}}\right)^{1 / 2}-1\right], \quad n=1,2, \cdots
\end{aligned}
$$


To simplify the discussion in this paper, the only quantum number addressed is $n$.

Next, when the part of Formula (29a) in parentheses is expressed as a Taylor expansion,

$$
\begin{gathered}
E_{\mathrm{re}, n} \approx m_{\mathrm{e}} c^{2}\left[\left(1-\frac{\alpha^{2}}{2 n^{2}}+\frac{3 \alpha^{4}}{8 n^{4}}-\frac{5 \alpha^{6}}{16 n^{6}}\right)-1\right] \\
\approx-\frac{\alpha^{2} m_{\mathrm{e}} c^{2}}{2 n^{2}} .
\end{gathered}
$$

From this, it is evident that Formula (15) derived by Bohr is an approximation of Formula (29).

Next, the following table summarizes the energies of a hydrogen atom obtained from Formulas (15) and (29). (Table 1) (Suto, 2020d)

Table 1. Comparison of the energies of a hydrogen atom predicted by Bohr's classical quantum theory and this paper

\begin{tabular}{ccc}
\hline$n$ & Bohr's Energy Levels, Formula (15) & This Paper, Formula (29) \\
\hline 1 & $-13.60569 \mathrm{eV}$ & $-13.60515 \mathrm{eV}$ \\
\hline 2 & $-3.40142 \mathrm{eV}$ & $-3.40139 \mathrm{eV}$ \\
\hline 3 & $-1.511744 \mathrm{eV}$ & $-1.511737 \mathrm{eV}$ \\
\hline
\end{tabular}

The following values of CODATA were used when calculating energies.

$$
\begin{gathered}
\alpha=7.2973525693 \times 10^{-3} . \\
c=2.99792458 \times 10^{-8} \mathrm{~m} \cdot \mathrm{s}^{-1} . \\
m_{\mathrm{e}}=9.1093837015 \times 10^{-31} \mathrm{~kg} .
\end{gathered}
$$

The following figures are the energy levels of the hydrogen atom derived by Bohr, and the energy levels derived by this paper (Figure 1). (Suto, 2021)

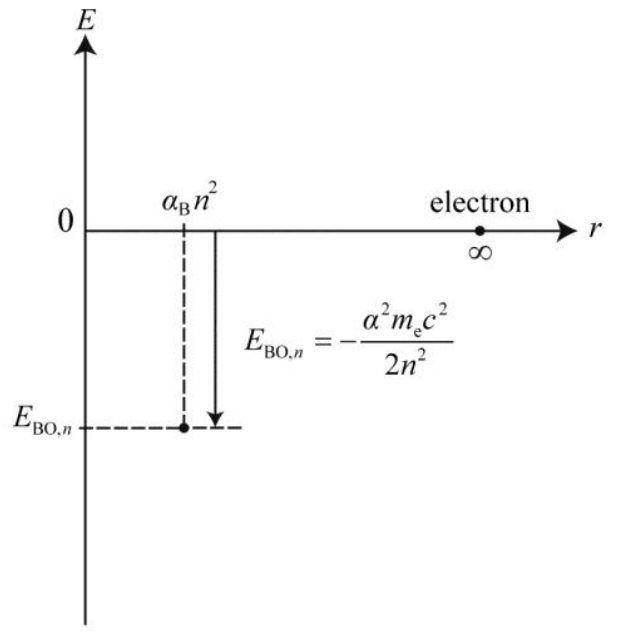

Figure 1a

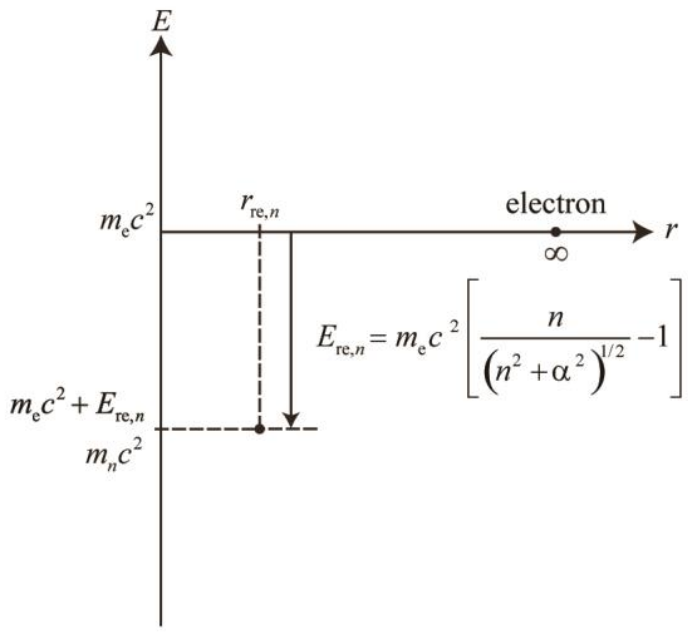

Figure 1b

Figure 1. (a) In Bohr's theory, the energy when the electron is at rest at a position infinitely distant from the proton (atomic nucleus) is defined to be zero. Formula (15) does not give energy levels derived from an absolute scale. 
(b) According to the STR, the energy of an electron at rest at a position where $r=\infty$ is $m_{\mathrm{e}} c^{2} . E_{\mathrm{re}, n}$ is given by the difference between $m_{\mathrm{e}} c^{2}$ and $m_{n} c^{2}$. In quantum mechanics, the lowest energy level of a hydrogen atom is $E_{1}$. However, Formula (11) can not only predict the energy levels of an ordinary hydrogen atom, derived by Bohr, but can also predict the existence of ultra-low energy levels.

\section{Orbital Radius of an Electron at the Negative Energy Level in Formula (28)}

Incidentally, there are also positive and negative solutions for $E_{\mathrm{ab}, n}\left(m_{n}\right)$ in Formula (28). Here, the ordinary, known energies of a hydrogen atom are expressed as $E_{\mathrm{ab}, n}^{+}, E_{\mathrm{re}, n}^{+}$. Also, the negative energies are expressed as $E_{\mathrm{ab}, n}^{-}, E_{\mathrm{re}, n}^{-}$.

The equation for positive energies is as follows.

$$
\begin{gathered}
E_{\mathrm{ab}, n}^{+}=m_{n} c^{2}=m_{\mathrm{e}} c^{2}+E_{\mathrm{re}, n}^{+} \\
=m_{\mathrm{e}} c^{2}\left(\frac{n^{2}}{n^{2}+\alpha^{2}}\right)^{1 / 2} .
\end{gathered}
$$

In contrast, the equations for the negative solutions are as follows.

$$
\begin{gathered}
E_{\mathrm{ab}, n}^{-}=-m_{n} c^{2}=-m_{\mathrm{e}} c^{2}-E_{\mathrm{re}, n}^{+} \\
=-m_{\mathrm{e}} c^{2}\left(\frac{n^{2}}{n^{2}+\alpha^{2}}\right)^{1 / 2} . \\
E_{\mathrm{re}, n}^{-}=-2 m_{\mathrm{e}} c^{2}-E_{\mathrm{re}, n}^{+} .
\end{gathered}
$$

If the relativistic mass of the electron is taken to be $m_{n}$, then the negative solution can be written as follows:

$$
m_{n}^{-}=-m_{\mathrm{e}}\left(1+\frac{\alpha^{2}}{n^{2}}\right)^{-1 / 2}<0 .
$$

A hydrogen atom in the $E_{\mathrm{ab}, n}^{-}$state will henceforth be called a "dark hydrogen atom" (DHA) in this paper.

Incidentally, the energy of the hydrogen atom can also be written as follows.

$$
E_{\mathrm{re}, n}=\frac{1}{2} V\left(r_{n}\right)=-\frac{1}{2} \frac{1}{4 \pi \varepsilon_{0}} \frac{e^{2}}{r_{n}}=-\frac{1}{2} m_{\mathrm{e}} c^{2} \frac{r_{\mathrm{e}}}{r_{n}}=-m_{\mathrm{e}} c^{2}\left(\frac{r_{\mathrm{e}} / 2}{r_{n}}\right) .
$$

Here, $r_{\mathrm{e}}$ is the classical electron radius as follows.

$$
r_{\mathrm{e}}=\frac{e^{2}}{4 \pi \varepsilon_{0} m_{\mathrm{e}} c^{2}}=2.8179403227 \times 10^{-15} \mathrm{~m} .
$$

Also, the following equation for energy can be obtained from Formula (35). 


$$
E_{\mathrm{ab}, n}^{+}=m_{n} c^{2}=m_{\mathrm{e}} c^{2}+E_{\mathrm{re}, n}^{+}=m_{\mathrm{e}} c^{2}\left(1-\frac{r_{\mathrm{e}} / 2}{r_{n}}\right)
$$

Here, if $-m_{\mathrm{e}} c^{2}$ is substituted for $E_{\mathrm{re}, n}$ in Formula (35), then the $r$ where $E_{\mathrm{ab}}=0$ is:

$$
r_{0}=\frac{r_{\mathrm{e}}}{2} .
$$

The radius $r$ where $E_{\mathrm{ab}}=0$ is $r_{\mathrm{e}} / 2$ due to Formula (37). Dirac predicted that the vacuum energy $E$ satisfies the relation $E<-m_{\mathrm{e}} c^{2}$, but actually $E_{\mathrm{ab}}=0$ is the energy of the virtual electron-positron pair which make up the vacuum (Figure 2). (Suto, 2017a).

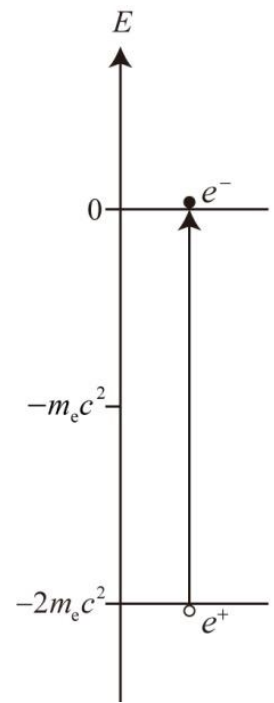

Figure 2a

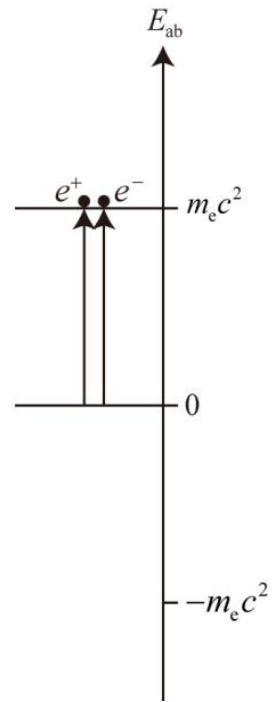

Figure $2 b$

Figure 2. Differences between Dirac's hole theory and the interpration in this paper

In Dirac's hole theory, when the $\gamma$-ray gives all of its energy to the virtual particles $\left(E=-2 m_{\mathrm{e}} c^{2}\right)$ comprising the vacuum around the atomic nucleus, a virtual particle acquires rest mass, and is emitted as an electron into free space, while the hole opened in the vacuum is the positron (Figure $2 \mathrm{a}$ ).

In the author's interpretation, an electron-positron pair is created because a $\gamma$-ray with an energy of $1.022 \mathrm{MeV}$ gives rest mass to a virtual electron-positron pair at the position $r=r_{\mathrm{e}} / 2$ (Figure 2b).

Dirac pointed out that there is a negative solution to Formula (1). Adopting the same viewpoint, there is a negative solution to Formula (11). To find the negative solution, it is necessary to create a quadratic equation for $r$. Thus, from Formulas (31b) and (37), (Suto, 2017a).

$$
\left(\frac{r_{n}-r_{\mathrm{e}} / 2}{r_{n}}\right)^{2}=\frac{n^{2}}{n^{2}+\alpha^{2}}
$$

From this, the following quadratic equation is obtained.

$$
r_{n}^{2}-\left(\frac{n^{2}+\alpha^{2}}{\alpha^{2}}\right) r_{\mathrm{e}} r_{n}+\left(\frac{n^{2}+\alpha^{2}}{\alpha^{2}}\right) \frac{r_{\mathrm{e}}^{2}}{4}=0 .
$$


If this equation is solved for $r_{n}$,

$$
r_{n}^{ \pm}=\frac{r_{\mathrm{e}}}{2}\left(1+\frac{n^{2}}{\alpha^{2}}\right)\left[1 \pm\left(1+\frac{\alpha^{2}}{n^{2}}\right)^{-1 / 2}\right]
$$

Next, if the electron orbital radii corresponding to the energy levels in Formulas (29) and (33) are taken to be, respectively, $r_{n}^{+}$and $r_{n}^{-}$,

$$
\begin{aligned}
& r_{n}^{+}=\frac{r_{\mathrm{e}}}{2} \frac{\left(n^{2}+\alpha^{2}\right)^{1 / 2}}{\left(n^{2}+\alpha^{2}\right)^{1 / 2}-n} \\
& r_{n}^{-}=\frac{r_{\mathrm{e}}}{2} \frac{\left(n^{2}+\alpha^{2}\right)^{1 / 2}}{\left(n^{2}+\alpha^{2}\right)^{1 / 2}+n} .
\end{aligned}
$$

Also, Formulas (42) and (43) can be written as follows. (Suto, 2017a)

$$
\begin{aligned}
& r_{n}^{+}=\frac{r_{\mathrm{e}}}{2}\left[1+\frac{n}{\left(n^{2}+\alpha^{2}\right)^{1 / 2}-n}\right] . \\
& r_{n}^{-}=\frac{r_{\mathrm{e}}}{2}\left[1-\frac{n}{\left(n^{2}+\alpha^{2}\right)^{1 / 2}+n}\right] .
\end{aligned}
$$

The next compares the orbital radii of an electron in a hydrogen atom $r_{n}^{+}$and the orbital radii of an electron with a negative mass $r_{n}^{-}$.

The following ratio is obtained from Formulas (42) and (43).

$$
\frac{r_{n}^{-}}{r_{n}^{+}}=\frac{\left(n^{2}+\alpha^{2}\right)^{1 / 2}-n}{\left(n^{2}+\alpha^{2}\right)^{1 / 2}+n}
$$

Here, if we set $n=1$,

$$
\frac{r_{1}^{-}}{r_{1}^{+}}=\frac{\left(1+\alpha^{2}\right)^{1 / 2}-1}{\left(1+\alpha^{2}\right)^{1 / 2}+1}=1.3312484168 \times 10^{-5} \approx \frac{1}{75120}
$$

Suto pointed out that an electron with negative mass forming DHA exists near the atomic nucleus (proton) (Suto, 2015; Suto, 2017b). A single DHA has about the same mass as an ordinary hydrogen atom. However, a DHA is far smaller than an ordinary hydrogen atom, and thus dark hydrogen can attain high density. Therefore, dark matter can be an important source of gravity.

If the above is indicated graphically, the result is as follows (Figure 3). (Suto, 2018). 


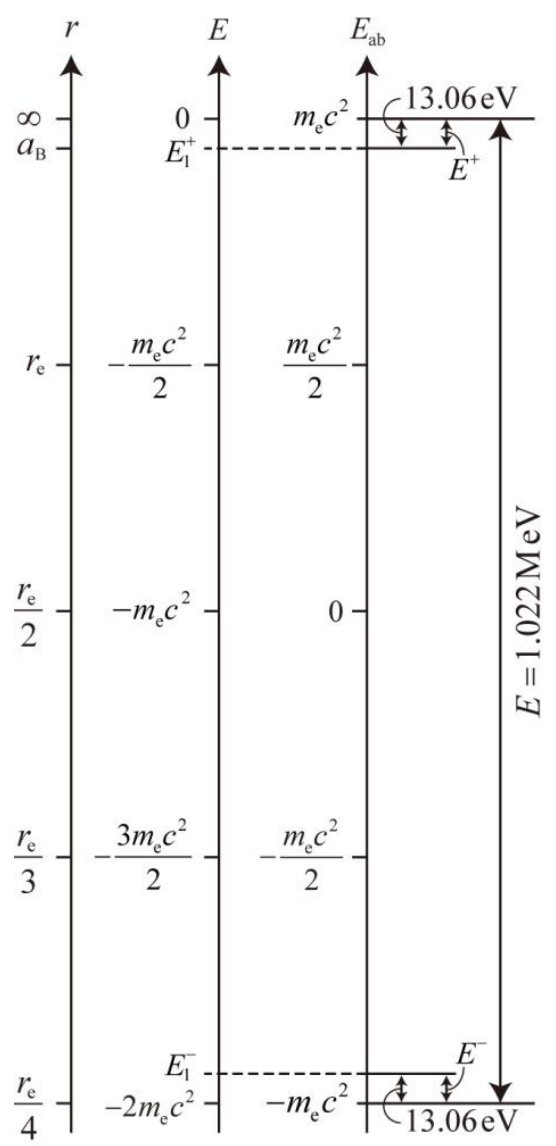

Figure 3. The energy levels of the hydrogen atom predicted by classical quantum theory $E_{n}^{+}\left(E_{\mathrm{ab}, n}^{+}\right)$and the new energy levels whose existence has been indicated by this paper $E_{n}^{-}\left(E_{\mathrm{ab}, n}^{-}\right)$

\section{Experiments Demonstrating the Existence of DHA}

How can hydrogen atoms in this energy state be verified? This paper looks at triplet production.

It is generally assumed that in triplet production, in which 2 electrons and 1 positron are created, electron-pair creation occurs not near the atomic nucleus, but near the electron in the outer shell orbital. A total of three particles are created in this case: one outer shell electron forming the atom, and a positron and electron created through pair production. However, in this model, $\left(1.022 \mathrm{MeV}-E_{n}^{+}\right)$should be sufficient as the necessary photon energy.

If an energy of $2.044 \mathrm{MeV}\left(4 m_{\mathrm{e}} c^{2}\right)$, is needed for triplet production, then the recoiled electron should be regarded as being at an ultra-low energy level.

Now, consider the case where an incident $\gamma$-ray has the energy corresponding to the mass of 4 electrons (2.044 $\mathrm{MeV}$ ). If this is discussed classically, the $\gamma$-ray can create an electron and positron near $r=r_{\mathrm{e}} / 2$ (Figure 4). (Suto, 2017b). 


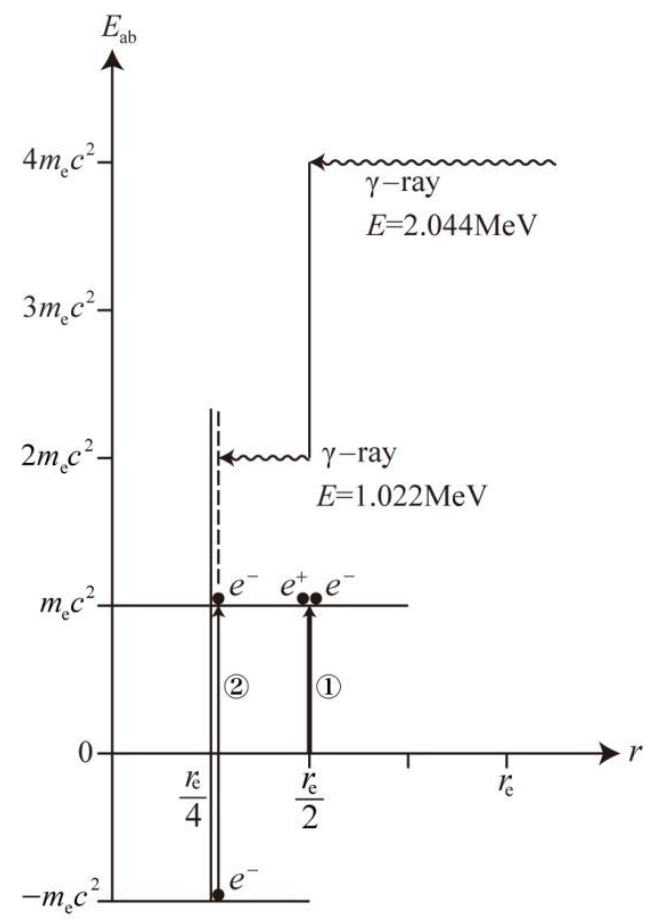

Figure 4. Interpretation of this paper regarding triplet production

Consider the case where a $\gamma$-ray with the energy of 4 electrons $(2.044 \mathrm{MeV})$ is incident on an atomic nucleus (proton).This $\gamma$-ray will give $1.022 \mathrm{MeV}$ of energy to the virtual particles at $r=r_{\mathrm{e}} / 2$, and an electron-positron pair will be created ( $\uparrow(1)$ ). When this $\gamma$-ray approaches closer to the atomic nuclear, and the electron in the orbital around the proton absorbs this energy, the electron will be excited and appear in free space ( $\uparrow(2)$ ). As a result, 2 electrons and 1 positron will appear in free space.

This paper points out that one of the two electrons which appears is an electron in the $E_{\mathrm{ab}, n}^{-}$state.

\section{Conclusion}

The hydrogen atom has ultra-low energy levels. In this state, there is an electron with negative mass near the atomic nucleus (proton) which has positive mass. Matter formed from this proton and an electron is DHA. This paper has focused on the triplet production experiment as the experiment demonstrating the existence of DHA. This paper concludes that one of the two electrons produced in triplet production is an electron which forms DHA. If the DM model advocated by this paper is correct, then DM has already been verified by experiment.

Is it possible to experimentally determine the correctness of previous explanations and the interpretation of this paper (Figure 4) regarding triplet production?

If four electrons can be produced in experiments using $\gamma$-rays with an energy of $2.044 \mathrm{MeV}\left(4 m_{\mathrm{e}} c^{2}\right)$, the interpretation of this paper is definitely superior. What causes this quadruplet production is an orbital electron, a dark electron which forms DHA, and a virtual electron and virtual positron which make up the vacuum. That is, the breakdown of the four produced particles is: 3 electrons and 1 positron.

Even if a quadruplet production phenomenon is rarely mixed in among the innumerable triplet production phenomena, the probability is likely to be extremely low. However, if the orbital electron is made to recoil at the stage before the $\gamma$-ray produces the electron-positron pair, then it should be possible to detect the quadruplet production phenomenon.

\section{Acknowledgments}

I would like to express my thanks to the staff at ACN Translation Services for their translation assistance. Also, I wish to express my gratitude to Mr. H. Shimada for drawing figures. 


\section{References}

Bohr, N. (1913). On the Constitution of Atoms and Molecules. Philosophical Magazine, 26, 1. https://doi.org/10.1080/14786441308634955

Bohr, N. (1952). (Collected Works Vol. 2). North-Holland, Amsterdam, 136.

Einstein, A. (1961). Relativity. Crown, New York, 43.

Sommerfeld, A. (1923). Atomic Structure and Spectral Lines. London: Methuen \& Co. Ltd, 528.

Suto, K. (2011). An Energy-momentum Relationship for a Bound Electron inside a Hydrogen Atom. Physics Essays, 24(2), 301-307. https://doi.org/10.4006/1.3092779

Suto, K. (2015). Presentation of Strong Candidates for Dark Matter. Global Journal of science frontier research: A, 15(7), 1-6.

Suto, K. (2017a). Region of Dark Matter Present in the Hydrogen Atom. Journal of Physical Mathematics, 8(4), 1000252.

Suto, K. (2017b). Presentation of Dark Matter Candidates. Applied Physics Research, 9(1), 70-76. https://doi.org/10.5539/apr.v9n1p70

Suto, K. (2018). Potential Energy of the Electron in a Hydrogen Atom and a Model of a Virtual Particle Pair Constituting the Vacuum. Applied Physics Research, 10(4), 93-101. https://doi.org/10.5539/apr.v10n4p93

Suto, K. (2019). The Relationship Enfolded in Bohr's Quantum Condition and a Previously Unknown Formula for Kinetic Energy. Applied Physics Research, 11(1), 19-34. https://doi.org/10.5539/apr.v11n1p19

Suto, K. (2020a). Dark Matter and the Energy-Momentum Relationship in a Hydrogen Atom. Journal of High Energy Physics, Gravitation and Cosmology, 6, 52-61. https://doi.org/10.4236/jhepgc.2020.61007

Suto, K. (2020b). Theoretical Prediction of Negative Energy Specific to the Electron. Journal of Modern Physics, 11, 712-724. https://doi.org/10.4236/jmp.2020.115046

Suto, K. (2020c). The Planck Constant Was Not a Universal Constant. Journal of Applied Mathematics and Physics, 8, 456-463. https://doi.org/10.4236/jamp.2020.83035

Suto, K. (2020d). Relativistic Correction of the Rydberg Formula. Journal of Modern Physics, 11, 294-303. https://doi.org/10.4236/jmp.2020.112018

Suto, K. (2021). The Quantum Condition That Should Have Been Assumed by Bohr When Deriving the Energy Levels of a Hydrogen Atom. Journal of Applied Mathematics and Physics, 9, 1230-1244. https://doi.org/10.4236/jamp.2021.96084

Zwicky, F (1933). Helv. Phys. Acta, 6, 110.

\section{Copyrights}

Copyright for this article is retained by the author(s), with first publication rights granted to the journal.

This is an open-access article distributed under the terms and conditions of the Creative Commons Attribution license (http://creativecommons.org/licenses/by/4.0/). 